\section{Endoscopic ultrasound-guided antegrade bile duct stone treatment followed by direct peroral trans- hepatic cholangioscopy in a patient with Roux-en-Y reconstruction}

Endoscopic ultrasound (EUS)-guided antegrade treatment for biliary disorders was developed for patients with an altered anatomy [1-5]. This report describes a case of successful EUS-guided bile duct stone (BDS) treatment followed by direct peroral transjejunal-hepatic cholangioscopy in a patient with Roux-en-Y reconstruction.

An 80-year-old woman with a BDS and a history of total gastrectomy with Rouxen-Y reconstruction was admitted to the Hokkaido University Hospital. The papilla could not be reached even with balloon enteroscopy. Therefore, transhepatic EUSguided antegrade BDS treatment was attempted.

A B3 branch duct was punctured using a 19-gauge needle (SonoTip Pro Control; Medi-Globe GmbH, Rosenheim, Germany), and a 0.025-inch guidewire (VisiGlide 2; Olympus Medical Systems, Tokyo, Japan) was placed ( Video 1). A 6-Fr wireguided diathermic dilator (Cysto-GastroSet; Endo-Flex GmbH, Voerde, Germany) was used to dilate the tract. Papillary balloon dilation (Hurricane RX Biliary Balloon Dilatation Catheter; Boston Scien- tific Japan, Tokyo, Japan) was also performed under fluoroscopic guidance according to the size of the distal bile duct ( $\bullet$ Fig. 1 a, $\bullet$ Video 1 ). The retrieval balloon (Extractor Pro RX retrieval balloon catheter, $15-18 \mathrm{~mm}$; Boston) and mechanical lithotripter (Litho Crush V, BML-V437QR-30; Olympus) both failed to extract the stone ( Video 1). A $6-\mathrm{Fr}$ nasobiliary drainage catheter (NBDC; Flexima ENBD Catheter; Boston Scientific Japan) was placed across the papilla for drainage into the duodenum and to facilitate a rendezvous procedure using balloon enteroscopy ( $\bullet$ Fig. 1 b).

The next day, the patient developed acute cholangitis. Re-intervention through the fistula tract was attempted. After advancing the guidewire into the bile duct, the BDS was captured by a standard basket catheter (FG-V435P; Olympus) ( Fig. 1 c - Video 1). However, the basket catheter could not pass the papilla and was impacted instead ( $\bullet$ Video 1 ). Emergency, di-
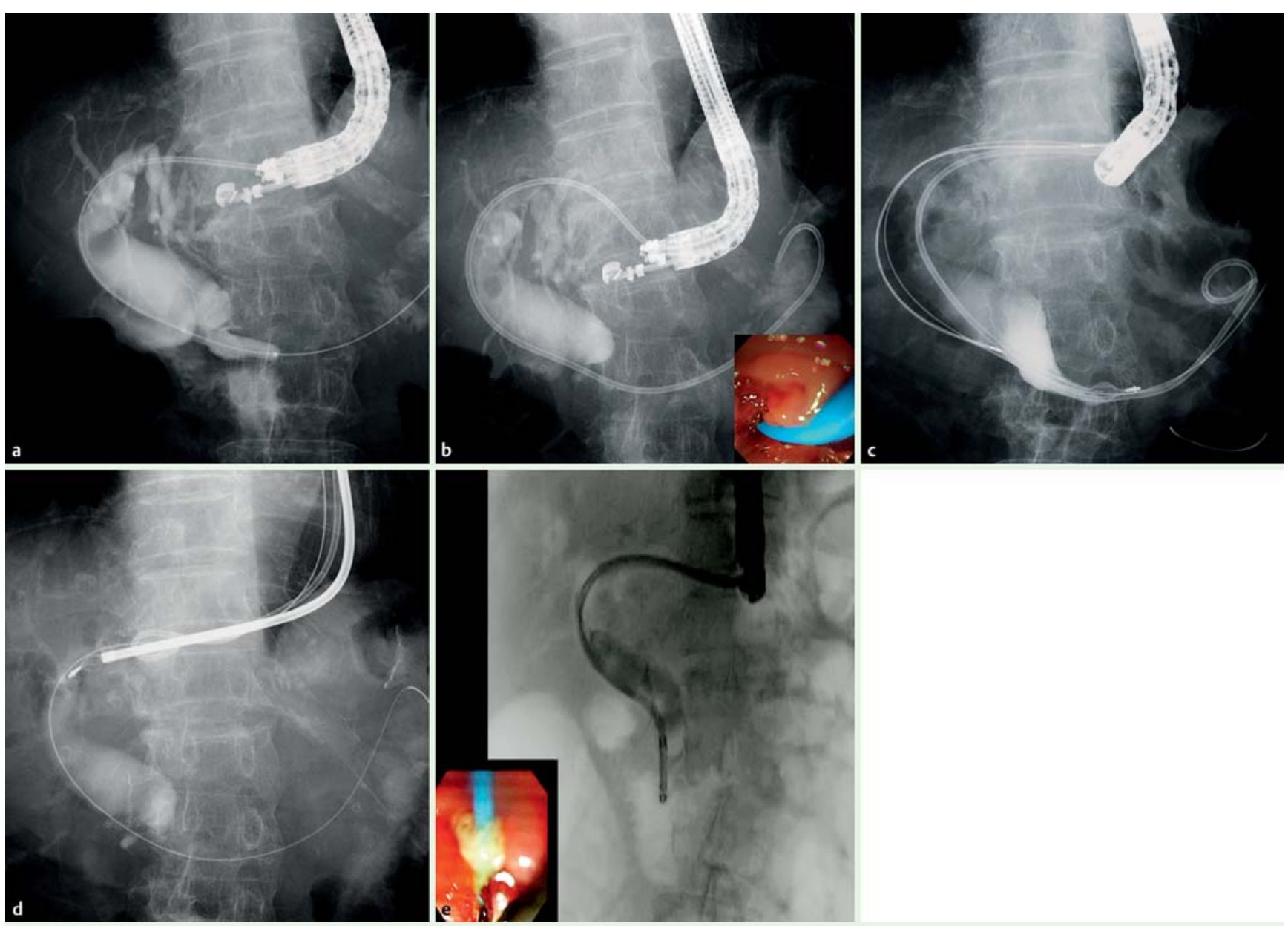

Fig. 1 Endoscopic ultrasound (EUS)-guided bile duct stone (BDS) treatment and direct, peroral, transjejunal-hepatic cholangioscopy in a patient with Rouxen-Y reconstruction. a Radiograph showing endoscopic ultrasound-guided standard papillary balloon dilation under fluoroscopic guidance. $\mathbf{b}$ Radiograph showing an endoscopic ultrasound-guided nasobiliary drainage catheter placed across the papilla. Inset: endoscopic image. c Radiograph showing a basket catheter that failed to advance across the papilla. $\mathbf{d}$ Radiograph showing an impacted bile duct stone crushed using peroral direct lithotripsy. e Radiograph showing direct, peroral, transhepatic cholangioscopy after endoscopic ultrasound-guided biliary drainage. 


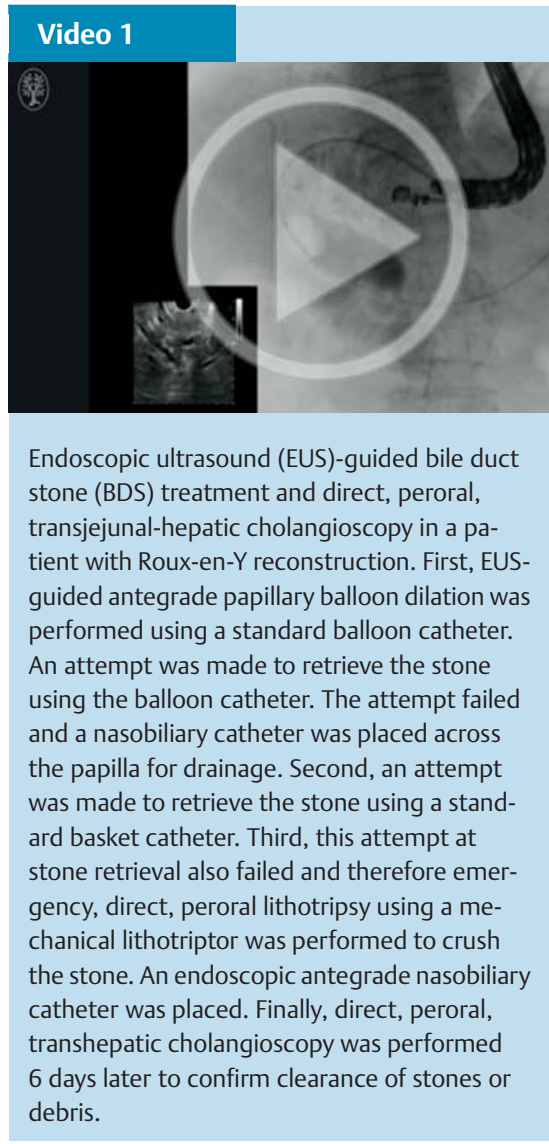

rect, peroral lithotripsy (BML-110A-1; Olympus) was performed ( $\bullet$ Fig. 1 d, - Video 1). After the BDS had been crushed, the NBDC was inserted into the bile duct without complications. At follow-up 6 days later, direct, peroral transhepatic cholangioscopy (CHF-B260, working channel $1.2 \mathrm{~mm}$; Olympus) was performed under therapeutic duodenoscope (TJF-260V; Olympus) guidance, and com- plete BDS clearance was confirmed (৫ Fig. 1 e, $\bullet$ Video 1 ).

An EUS-guided rendezvous procedure is generally performed when EUS-guided antegrade BDS treatment fails. However, endoscopic re-intervention through the fistula tract should be considered in patients with altered gastrointestinal anatomy. To our knowledge, this is the first report of a troubleshooting technique for BDS impaction using direct, peroral, mechanical lithotripsy and confirmation of BDS clearance by direct antegrade cholangioscopy following EUS-guided biliary drainage. Although challenging, this stone extraction technique combined with EUSguided antegrade cholangiography and cholangioscopy (EUS-guided ACC) should be recognized as a treatment for BDS in patients with altered gastrointestinal anatomy.

Endoscopy_UCTN_Code_TTT_1AS_2AD

Competing interests: None

Hiroshi Kawakami ${ }^{1}$, Masaki Kuwatani ${ }^{2}$, Yoshimasa Kubota ${ }^{1}$, Shuhei Kawahata', Kimitoshi Kubo ${ }^{1}$, Kazumichi Kawakubo $^{3}$, Naoya Sakamoto ${ }^{3}$

${ }^{1}$ Department of Gastroenterology and Hepatology, Hokkaido University Hospital, Sapporo, Japan

2 Division of Endoscopy, Hokkaido University Hospital, Sapporo, Japan

${ }^{3}$ Department of Gastroenterology and Hepatology, Hokkaido University Graduate School of Medicine, Sapporo, Japan

\section{References}

1 Weilert F, Binmoeller KF, Marson F et al. Endoscopic ultrasound-guided anterograde treatment of biliary stones following gastric bypass. Endoscopy 2011; 43: 1105-1108

2 Iwashita T, Yasuda I, Doi S et al. Endoscopic ultrasound-guided antegrade treatments for biliary disorders in patients with surgically altered anatomy. Dig Dis Sci 2013; 58: 2417-2422

3 Itoi T, Sofuni A, Tshichiya T et al. Endoscopic ultrasonography-guided transhepatic antegrade stone removal in patients with surgically altered anatomy: case series and technical review (with videos). J Hepatobiliary Pancreat Sci 2014; 21: E86-93

4 Kawakami H, Kuwatani M, Kawakubo Ket al. Endoscopic ultrasound-guided antegrade diathermic dilation followed by self-expandable metal stent placement for malignant distal biliary stricture. Endoscopy 2014; 46 (Suppl. 01): E328 -E329

5 Kawakami H, Kuwatani M, Sakamoto N Endoscopic ultrasound-guided antegrade diathermic dilation followed by self-expandable metallic stent placement for anastomotic stricture after hepaticojejunostomy (with video). Dig Endosc 2014; 26: $121-122$

\section{Bibliography}

Dol http://dx.doi.org/

10.1055/s-0034-1392507

Endoscopy 2015; 47: E340-E341

(c) Georg Thieme Verlag KG

Stuttgart · New York

ISSN 0013-726X

\section{Corresponding author}

Hiroshi Kawakami, MD, PhD

Department of Gastroenterology and Hepatology Hokkaido University Hospital

Kita 14, Nishi 5, Kita-ku

Sapporo 060-8648

Japan

Fax: +81-11-7067867

hiropon@med.hokudai.ac.jp 